\title{
Adipocyte Secretome Increases Radioresistance of Malignant Melanocytes by Improving Cell Survival and Decreasing Oxidative Status
}

\author{
Pedro Coelho, ${ }^{a, b, c, 1}$ Liliana Silva,${ }^{b, c, 1}$ Isabel Faria, ${ }^{d}$ Mónica Vieria, ${ }^{b, c}$ Armanda Monteiro, ${ }^{e}$ Gabriela Pinto, ${ }^{e}$ Cristina \\ Prudêncio, ${ }^{b, c}$ Rúben Fernandes ${ }^{b, c}$ and Raquel Soares ${ }^{a, b, 2}$
}

\begin{abstract}
${ }^{a}$ Department of Biochemistry, Faculty of Medicine and ${ }^{b}$ Instituto de Inovação e Investigação em Saúde, University of Porto, Portugal; ${ }^{c}$ Ciências Químicas e Biomoléculas, CISA and "Radioterapia, Escola Superior de Tecnologia da Saúde do Porto, Instituto Politécnico do Porto, Portugal; and - Serviço de Radioterapia, Centro Hospitalar de São João, Porto, Portugal
\end{abstract}

Coelho, P., Silva, L., Faria, I., Vieira, M., Monteiro, A., Pinto, G., Prudêncio, C., Fernandes, R. and Soares, R. Adipocyte Secretome Increases Radioresistance of Malignant Melanocytes by Improving Cell Survival and Decreasing Oxidative Status. Radiat. Res. 187, 000-000 (2017).

Radiotherapy is a treatment option for the majority of malignancies. However, because melanoma is known to be radioresistant, the use of ionizing radiation as an adjuvant therapy in cutaneous melanoma patients is ineffective. Obesity has now been recognized as a risk factor for melanoma. High adiposity is generally associated with a more pro-oxidative status. Oxidative stress is a major player in radiation therapy and also a common link between obesity and cancer. Several adipocyte-released proteins are known to have a role in controlling cellular growth and pro-survival signaling. For that reason, we investigated the influence of 3T3-L1 mature adipocyte secretome in B16-F10 malignant melanocyte radiosensitivity. We evaluated B16-F10 cell survival and redox homeostasis when exposed to four daily doses of ionizing radiation ( $2 \mathrm{~Gy}$ per day) up to a total of $8 \mathrm{~Gy}$ in a medical linear accelerator. B16-F10 melanocytes exhibited slight alterations in survival, catalase activity, nitrative stress and total oxidant concentration after the first 2 Gy irradiation. The motility of the melanocytes was also delayed by ionizing radiation. Subsequent irradiations of the malignant melanocytes led to more prominent reductions in overall survival. Remarkably, 3T3-L1 adipocyte-secreted molecules were able to increase the viability and migration of melanocytes, as well as lessen the pro-oxidant burden induced by both the single and cumulative X-ray doses. In vitro adipocyte-released factors protected B16-F10 malignant melanocytes from both oxidative stress and loss of viability triggered by radiation, enhancing the radioresistant phenotype of these cells with a concomitant activation of the AKT signaling pathway. These results both help to elucidate how obesity influences melanoma radioresistance and support the usage of conventional medical linear accelerators as a valid

Editor's note. The online version of this article (DOI: 10.1667/ RR14551.1) contains supplementary information that is available to all authorized users.

2 Address for correspondence: Departamento de Bioquímica, Faculdade de Medicina da Universidade do Porto, Al. Prof. Hernâni Monteiro, 4200-319 Porto, Portugal; email: raqsoa@med.up.pt. model for the in vitro radiobiological study of tumor cell lines. (c) 2017 by Radiation Research Society

\section{INTRODUCTION}

Melanoma is one of the world's most rapidly increasing malignancies (1) The number of reported cases increasing for the last few decades. Over 176,000 new cases of melanoma are reported annually in Europe and the U.S. (2, 3). Melanoma treatment has remained the same for the last few decades: surgical excision of the malignant mass is still the most effective treatment in primary melanomas (4).

Radiotherapy is frequently used to treat the majority of malignancies, and has a direct impact in the proliferative phenotype of both normal and cancer cells. Radiationinduced ionization of regulatory proteins and DNA might render the cells unviable and culminate in cellular death (5). Ionizing radiation can also indirectly cause cellular damage. The formation of highly reactive oxygen and nitrogen radicals increases the intracellular oxidative stress, depleting the antioxidant defenses, which subsequently react with many cellular components (DNA, proteins, lipids) leading to unrecoverable damage (6).

However, melanoma is known to be radioresistant, which discourages the use of ionizing radiation as an adjuvant therapy in melanoma patients (7). Recently, the use of radiation in higher delivered doses, hypofractionated and in combination with immunotherapy has led to some positive outcomes in melanoma metastasis treatment and palliation $(8-10)$. Nonetheless, obesity, particularly high visceral adiposity, presents a problem in treatment planning and delivery of radiation to internal metastases. Generally, higher body adiposity is associated with both cancer initiation and progression (11). Obesity itself is a risk factor for several types of neoplasms, including melanomas (12, 13). High adiposity can be a contraindication for (and may limit the extent of) cancer surgery, since it contributes to the 
inadequate dosing of chemotherapeutic drugs and complicates the planning and delivery of radiation (14-16).

Adipocytes secrete a variety of factors that exert effects at both local and systemic levels (17). The grand majority of these factors are cytokines, chemokines and inflammatory mediators, but a role in growth regulation as a new aspect of adipokines has been revealed by novel adipocyte-released molecules (18). Fat-derived molecules stimulate melanoma progression and aggressiveness and act as mediators of proliferation in melanoma cells $(19,20)$.

Resistance to oxidative stress appears to be a key mechanism of tumor radioresistance (21). Obesity is linked to a more pro-oxidative status, with a concomitant systemic increase in reactive oxygen species (ROS), acting as an additional source of oxidants (22).

For the current study, we hypothesized that adipocytes might lead to two antagonistic outcomes towards melanocyte radiosensitivity. Although the fat-derived growth factors might protect melanocytes from radiation-induced loss of survival by stimulating their overall proliferation, the adipocyte-generated oxidants can further increase the oxidative burden, aggravating the radiation-induced damages. In this study, we irradiated cell cultures at standard doses to investigate the action of the adipocyte secretome in melanoma radioresistance.

\section{MATERIALS AND METHODS}

\section{Cell Cultures}

B16-F10 melanocytes and 3T3-L1 pre-adipocytes were maintained in high-glucose Dulbecco's modified Eagle medium (DMEM; SigmaAldrich ${ }^{\circledR}$ LLC, St. Louis, MO) supplemented with $10 \%$ fetal bovine serum (FBS) and $1 \%$ penicillin/streptomycin/amphotericin B (SigmaAldrich). Cells were culture at $37^{\circ} \mathrm{C}$ in an incubator with a $5 \% \mathrm{CO}_{2}$ humidified atmosphere.

B16-F10 melanocytes were cultured in 96-well plates $\left(1 \times 10^{4}\right.$ cells/ $\mathrm{ml})$ for viability determination and in 24-well plates $\left(10 \times 10^{4}\right.$ cells/ $\mathrm{ml})$ for the other assays. All treatments were performed in serum-free conditions and after a $16 \mathrm{~h}$ FBS-starving period.

\section{Adipocytes Differentiation and Conditioned Media Collection}

Two days after 3T3-L1 pre-adipocyte cultures reached confluence, a mixture of $2 \mu M$ insulin (Sigma-Aldrich), $1 \mu M$ dexamethasone (Sigma-Aldrich) and $0.25 \mathrm{~m} M$ isobutylmethylxanthine (Fluka ${ }^{\mathrm{TM}} /$ Honeywell Research Chemicals, Morris Plains, NJ) was added to the cultures. The media was replaced and the cells maintained with 2 $\mu M$ insulin every two to three days. On day 7 after induction, cultures were washed with phosphate buffered saline (PBS) and incubated in serum-free DMEM. On the next day, the conditioned media, enriched with adipocyte secretome, was harvested from the adipocyte cultures, centrifuged for $5 \mathrm{~min}$ at $300 \mathrm{~g}$ and the supernatant was collected for the subsequent treatments.

\section{Irradiation of Cells}

Prior to irradiation, computed tomography scans were performed to obtain three-dimensional (3D) images and calculate the density of both the 24- and 96-well plates. To simulate a biological structure, the plates were placed between two 5-cm-height water phantoms (Supplementary Fig. S1; http://dx.doi.org/10.1667/RR14551.1.S1).
The 3D conformal radiation dosimetric plan comprised two fields (one anteroposterior and one posteroanterior). Irradiations were performed using the software XIO-Release version 4.70.02 (Supplementary Fig. S2; http://dx.doi.org/10.1667/RR14551.1.S1). A total radiation dose of $8 \mathrm{~Gy}$ in four daily fractions of $2 \mathrm{~Gy}$, prescribed to the isocenter, was delivered in a PRIMUS ${ }^{\mathrm{TM}}$ linear accelerator (Siemens) with a $6 \mathrm{MV}$ photon beam.

\section{Metabolic Activity Assay}

The (4,5-dimethylthiazol-2-yl)-2,5-diphenyltetrazolium bromide (MTT; Sigma-Aldrich) was used to assess the metabolic activity of malignant melanocytes (B16-F10) irradiated under the influence of the adipocyte secretome (3T3-L1). B16-F10 cells were seeded in 96-well plates and cultured for $24 \mathrm{~h}$. Afterwards, cells were treated with the mature 3T3-L1 adipocyte conditioned media (CM) and irradiated. Next, $20 \mu \mathrm{l}$ of MTT was added to each well and incubated for $3 \mathrm{~h}$. After this period, the violet formazan precipitate was dissolved in 100 $\mu \mathrm{l}$ of dimethyl sulfoxide (Merck \& Co., Kenilworth, NJ) and the absorbance at 550 and $650 \mathrm{~nm}$ recorded. Metabolic activity was calculated by the formula $\mathrm{ABS}_{\text {(final) }}=\mathrm{ABS}_{(550)}-\mathrm{ABS}_{(650)}$, and normalized by dividing over the absorbance of the control.

\section{Total Antioxidant Status Determination}

The in vitro determination of the total antioxidant status (TAS) was done with the TAS kit (Randox Laboratories, Crumlin, UK) following the manufacturer's instructions. Briefly, $5 \mu \mathrm{l}$ of the sample were mixed with $200 \mu \mathrm{l}$ of the chromogenic solution and the substrate was added to a final volume of $250 \mu \mathrm{l}$. The appearance of the radical cation $\mathrm{ABTS}^{*+}$ was monitored for $15 \mathrm{~min}$ at $600 \mathrm{~nm}$. Antioxidants suppress the blue-green color production to a degree that is proportional to their concentrations. Calibration was performed using Trolox (6-hydroxy2,5,7,8-tetramethylchroman-2-carboxylic acid) standards and the results were expressed as $\operatorname{TAS}_{(\mathrm{mmol} / \mathrm{ml})}=\mid$ Trolox standards $\mid /$ $\left(\Delta \mathrm{ABS}_{\text {(samples) }}-\Delta \mathrm{ABS}_{\text {(Trolox) }}\right)$, where $\triangle \mathrm{ABS}=\mathrm{ABS}_{\text {final }}-\mathrm{ABS}_{\text {initial }}$, according to the manufacturer instructions.

\section{Catalase Activity}

Catalase activity was determined based on the rate of decomposition of $\mathrm{H}_{2} \mathrm{O}_{2}$, which is proportional to the reduction of the absorbance at $240 \mathrm{~nm}$. A volume of $100 \mu \mathrm{l}$ of each sample was added to $400 \mu \mathrm{l}$ of 5 $\mathrm{m} M$ hydrogen peroxide substrate solution in $0.05 M$ phosphate buffer $(\mathrm{pH} 7.0)$. The decay kinetics for absorbance was determined in a Jenway $6505 \mathrm{Uv} / \mathrm{Vis}$ spectrophotometer for $1 \mathrm{~min}$. Results are expressed as $|\Delta \mathrm{ABS} / \mathrm{min}| \times 10^{6}$.

\section{TBARS Determination}

To determine thiobarbituric acid-reactive substances (TBARS), 100 $\mu \mathrm{l}$ of thiobarbituric acid 10\% (w/v) (Merck \& Co) were added to 500 $\mu \mathrm{l}$ of cellular extract and placed in a boiling water bath for $30 \mathrm{~min}$. Afterwards, the amount of malondialdehyde (MDA) present was determined by measuring the absorbance at $535 \mathrm{~nm}$. The standard curve was performed with standard solutions of malonaldehyde bis(dimethyl acetal) (Sigma-Aldrich).

\section{Nitrotyrosine Quantification}

B16 cell lysates were prepared in PBS and sonicated for $10 \mathrm{~min}$ (SilentCrusher S; Heidolph North America, Elk Grove Village, IL). Proteins and other cellular components were precipitated with $15 \%$ (v/ v) ice-cold trifluoroacetic acid upon centrifugation at $10,000 \mathrm{rpm}$ for $10 \mathrm{~min}$. Chromatographic detection of 3-nitrotyrosine (3-NT) in the supernatants was performed as described by Teixeira et al. (23) (see Supplementary Table S1 for detailed chromatographic conditions; http://dx.doi.org/10.1667/RR14551.1.S1). 3-NT standards were pre- 
pared from lyophilized 3-Nitro-L-tyrosine (Santa Cruz Biotechnology ${ }^{\circledR}$ Inc., Dallas, TX). The reverse-phase HPLC VWR-Hitachi Elite LaChrom (VWR) chromatographic system that was used consisted of a quaternary pump, model HTA L-2130, an autosampler L-27200, a ODS-Hypersil C18 analytical column $(200 \mathrm{~cm} \times 4 \mathrm{~mm}$ i.d.; $5 \mu \mathrm{m}$ particle size) (Merck \& Co.) in a L-2300 column oven and a diode array detector, model L-2455. The resulting chromatograms (Supplementary Fig. S3; http://dx.doi.org/10.1667/RR14551.1.S1) were analyzed using Agilent EZChrom Elite software version 3.3.2 (Agilent Technologies Inc., Palo Alto, CA).

\section{In Vitro Scratch Assay}

Using a pipette tip, an injury was inflicted in B16-F10 confluent cultures. Cells were then incubated after 3T3-L1 conditioning and/or irradiation. At 4, 12 and $24 \mathrm{~h}$ after treatment, the migrated distance was photographed under an inverted microscope (Nikon ${ }^{\circledR}$ Instruments Inc., Melville, NY) at a $200 \times$ magnification and the scratch closure was determined by measuring the injury width with ImageJ software (National Institutes of Health, Bethesda, MD). Shown are the normalized values $( \pm \mathrm{SEM})$ of nine measurements.

\section{Western Blotting Analysis}

Proteins were extracted using RIPA buffer from B16 cell lysates. Equal volumes of protein extracts were loaded onto a $10 \%$ SDSPAGE with a 5\% stacking gel. After electrophoretic separation, proteins were blotted into a nitrocellulose membrane (Amersham Life Science Inc., Cleveland, OH). Immunodetection for total AKT, phosphorylated AKT (Ser473), and $\beta$-actin (all from Cell Signaling Technology ${ }^{\circledR}$ Inc., Danvers, MA) was accomplished with enhanced chemiluminescence (Clarity ${ }^{\mathrm{TM}}$ ECL kit; Bio-Rad ${ }^{\circledR}$ Laboratories Inc., Hercules, CA).

\section{Statistical Analysis}

Statistical analysis was performed using GraphPad Prism 6.0 (GraphPad Software Inc., LaJolla, CA). Differences between treatments were evaluated by Student's $t$ test or two-way ANOVA with Sidak multiple comparisons test accordingly to the number of conditions and treatments. Significance level was set to $P<0.05$.

\section{RESULTS}

\section{T3-L1 CM Protects B16-F10 Cells from Radiation Damage}

To access the susceptibility of B16-F10 cells to the direct effects of ionizing radiation, B16-F10 melanocytes were irradiated with a single $2 \mathrm{~Gy}$ dose of $\mathrm{X}$ rays and the cellular viability was then evaluated by the MTT assay. Upon irradiation, B16-F10 cells showed a slight (10\%) decrease in their metabolic activity. However, $12 \mathrm{~h}$ later the metabolic activity of the irradiated melanocytes was improved to control values (Fig. 1A). When cultured with 3T3-L1 CM the malignant melanocytes significantly increased their metabolic activity by more than $30 \%$ whether irradiated or not (Fig. 1A).

Ionizing radiation imbalances the cellular redox homeostasis (6). Accordingly, we next evaluated oxidative stress markers and antioxidant status of the irradiated B16-F10 melanocytes.

Lipid oxidation by ROS gives rise to a number of byproducts. MDA is one of the principal end products of fatty acid peroxidation in cells (24). We evaluated the MDA levels by the TBARS assay but found no significant differences in lipid peroxidation with either irradiation or 3T3-L1 secreted factors at the two time points studied (Fig. 1B).

Catalase is an oxidative stress protective enzyme that neutralizes hydrogen peroxide formed during oxidative stress. The activity of this enzyme in B16-F10 melanocytes was not altered after exposure to ionizing radiation. However, a significantly lower activity level was observed when cells were treated with 3T3-L1-secreted molecules (Fig. 1C). Incubation with adipocyte 3T3-L1 CM resulted in a significant increase in catalase activity in the melanocytes $4 \mathrm{~h}$ postirradiation.

Although the total antioxidant capacity of 3T3-L1 CM is significantly greater than fresh culture media (Fig. 1D), when fed to B16-F10 cultures and irradiated, the number of antioxidants present in the 3T3-L1 CM is no different from the control $12 \mathrm{~h}$ after treatment (Fig. 1E). Interestingly, the intracellular production of redox scavenger mechanisms is stimulated by the adipocyte secretome and counterbalances the lower radiation-induced antioxidant defense status (Fig. $1 \mathrm{~F})$.

To address the ability of melanocytes to migrate, a mechanical damage was inflicted to confluent melanocyte cultures and the scratch closure was microscopically inspected (Fig. 2B). Melanocytes rapidly occupied the injured area when incubated with 3T3-L1 CM. Exposure to radiation (2 Gy) slowed down B16-F10 motility towards the injury void space, particularly $4 \mathrm{~h}$ postirradiation, but this effect was reversed when 3T3-L1 CM was also present (Fig. 2A).

These results indicate that adipocyte-released molecules can protect melanoma cancer cells from X-ray radiation damages, both reducing antioxidant status and enhancing cell migration.

\section{Adipocyte Secretome Contributes to Melanoma Radioresistance}

As radiation dose increases, the amount of direct and indirect oxidative stress-mediated cellular damages increases proportionally. Our next approach was to determine the effects of cumulative radiation of B16-F10 melanocytes. A total dose of 8 Gy was delivered in four daily 2 Gy irradiations to melanoma cells in the presence of the 3T3-L1 adipocyte-derived factors.

The metabolic viability of the irradiated melanocytes displayed a constant decrease of approximately $20 \%$, for radiation doses greater than 4 Gy. However, 3T3-L1 CM treatment of B16-F10 cells significantly increased the survival of the melanocytes suppressing the radiationinduced cytotoxic effects (Fig. 3A). Catalase activity followed a similar trend: radiation promotes a diminished basal catalase activity but when in the presence of the 3T3L1 CM, catalase activity is highly enhanced (Fig. 3B). 

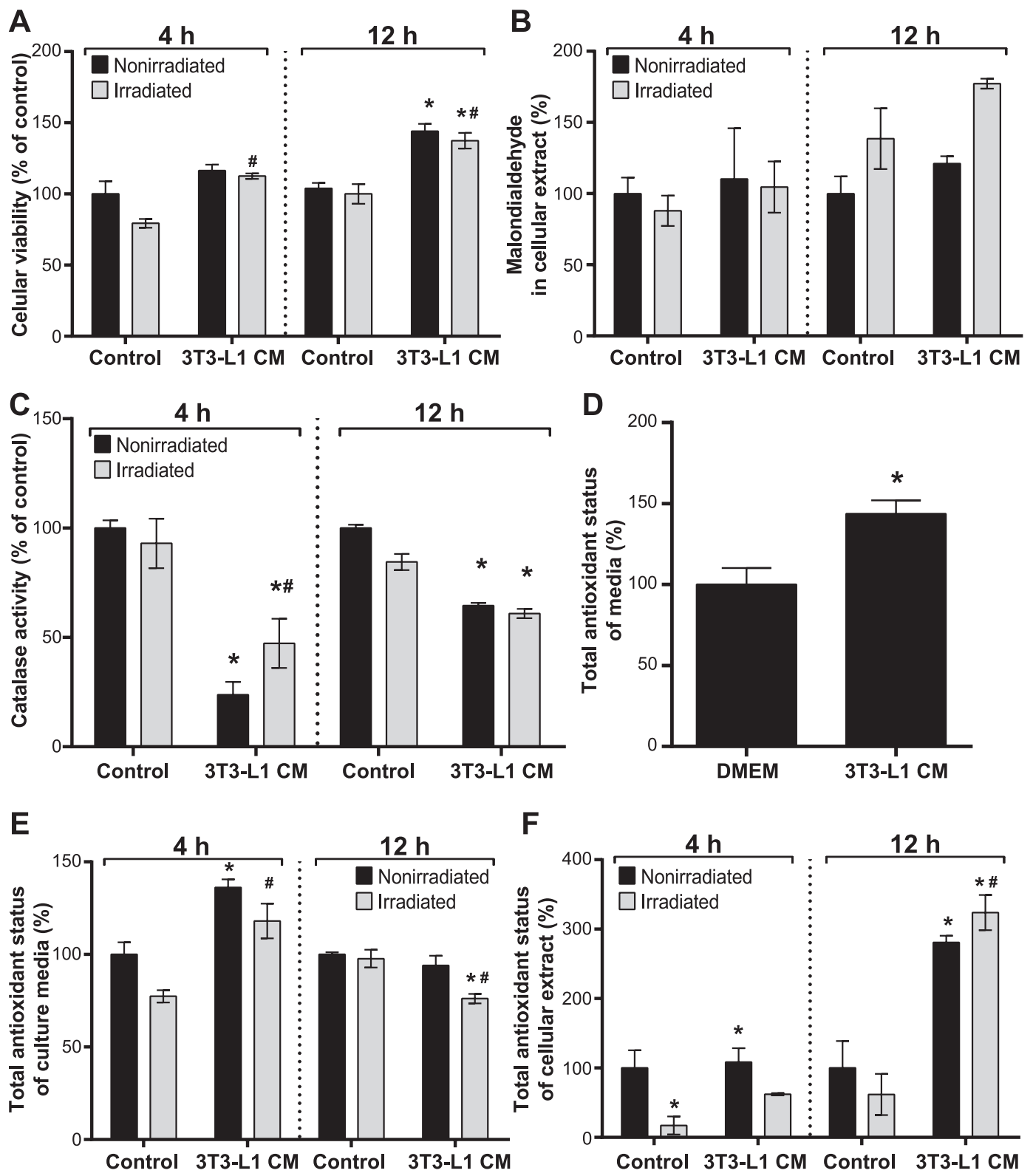

FIG. 1. B16-F10 survival and antioxidant status upon single 2 Gy irradiation and treated with 3T3-L1 CM. Controls were treated with serum-free DMEM. Panel A: Metabolic activity of melanoma cells by MTT reduction assay. Results represent the percentage of viable cells normalized by the absorbance of control. Panel B: Levels of MDA by TBARS determination in B16-F10 cell lysates. Panel C: Catalase activity was determined based on the reduction of absorbance at $240 \mathrm{~nm}$. Total antioxidant concentration in fresh 3T3-L1 CM and DMEM (panel D) and in the treated B16-F10 culture supernatant (panel E) and cellular extract (panel F) was determined enzymatically by the TAS assay. Shown are mean values ( \pm SEM) of three measurements for each time point/ condition. Results are normalized as a percentage of the control treatment ${ }^{*} P<0.05$ vs. control treatment; ${ }^{\#} P<$ 0.05 vs. irradiation).

Reactive nitrogen species (RNS) lead to oxidative protein modifications. Free 3-NT is a biomarker of the turnover of radiation-induced nitrated proteins (25). The levels of 3-NT rapidly increased with the first cumulative radiation doses, but by the end of the experiment the 3-NT levels approximated those of the control group. 3T3-L1released factors protected irradiated B16-F10 cells from oxidative nitration primarily in the first radiation fractions (Fig. 3C).
Activation of the PI3K-AKT signaling pathway has been correlated with radiation resistance $(26,27)$. To investigate whether these radioresistant inducer effects of 3T3-L1 CM were due to activation of the PI3K-AKT signaling pathway, immunoblotting for AKT and Ser473 phospho-AKT was performed in B16-cell lysates. The expression of the active form of AKT was only detected in treatment groups containing the 3T3-L1 CM (Fig. 3D), confirming the stimulation of this pathway by adipocyte-released factors. 

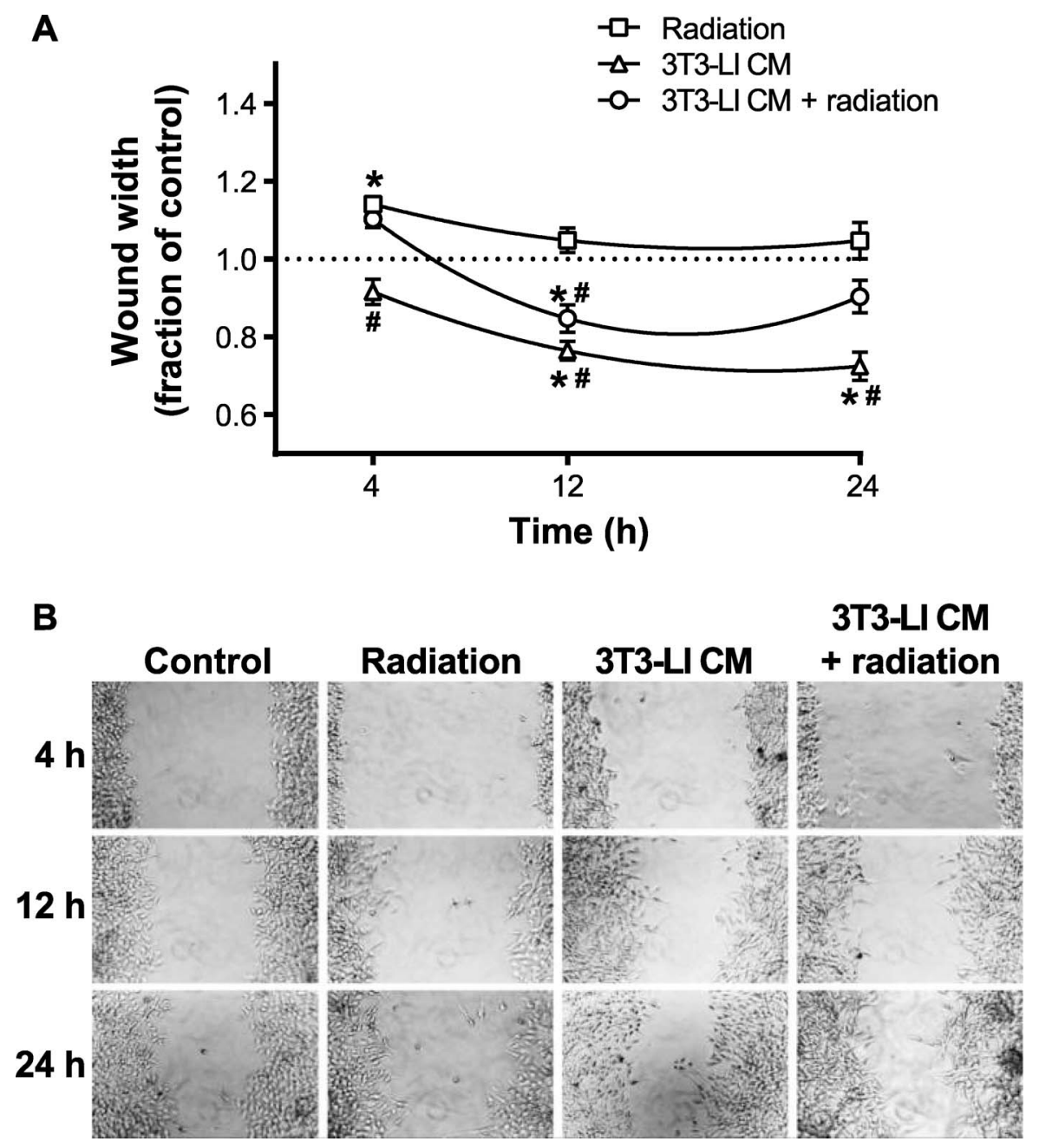

FIG. 2. Migration of B16-F10 melanocytes exposed to 3T3-L1 CM and/or radiation (2 Gy). Panel A: Injury closure fraction was determined by diving over the width of void space of the control. Bars show mean values $( \pm \mathrm{SEM})$ of nine measurements for each time point/condition. ( ${ }^{*} P<0.05$ vs. control treatment; ${ }^{*} P<0.05$ vs. irradiation). Panel B: Representative microscopy images of the injuries for each treatment and time point (200×).

These findings further underscore the protective effects of the adipocyte secretome in shielding melanocytes from oxidative stress and loss of viability triggered by repetitive fractions of ionizing radiation.

\section{DISCUSSION}

Cell survival response and (anti)oxidant status are good indicators of radiation susceptibility. Our results disclosed an increased metabolic activity of irradiated melanocytes when treated with adipocyte conditioning media (3T3-L1 CM). Low-dose irradiation (2 Gy) was only able to induce significant alterations in B16-F10 viability in the early hours after treatment. However, repeated irradiation of melanocytes, up to a total of $8 \mathrm{~Gy}$, led to a constant decrease in metabolic activity. On the other hand, when 3T3-L1adipocyte secreted molecules were present, radiationinduced loss of viability was overturned and melanocytes had higher metabolic activities.
Melanocytes are considered to be resistant to radiation damage (28). Ionizing radiation increases the oxidative burden in tumor cells (29). The mild decrease in catalase activity and antioxidant capacity combined with the temporary increase in protein nitrative stress observed corroborate the low radiosensitivity of B16-F10 melanocytes. Nevertheless, adipocyte-released factors enhanced the capacity of melanocytes to fight radiation-induced oxidative stress, further contributing to melanoma radioresistance.

In obese patients there is a shift in the delivery of external-beam radiation, resulting in the target location not receiving the full dose (30). In addition to the physical constraints to dose planning and delivery, our data further reinforce that the adipokine-rich environment might further contribute to the radioresistance of the melanocytes, culminating in a global underdosing effect. In addition to careful planning and dose delivery, which must take into account the higher body mass index of the patients, consideration must also be given to the more pro- 

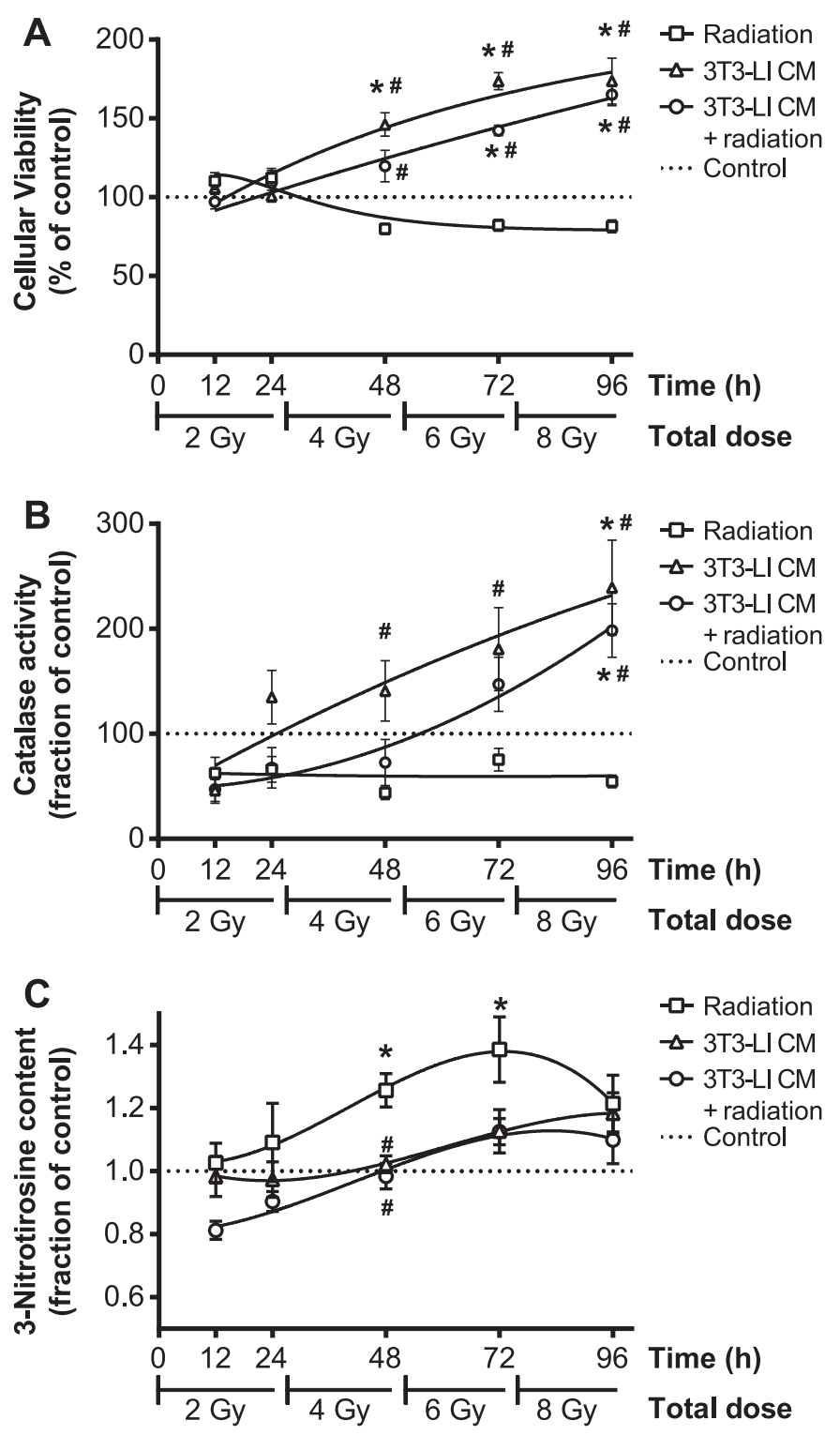

D

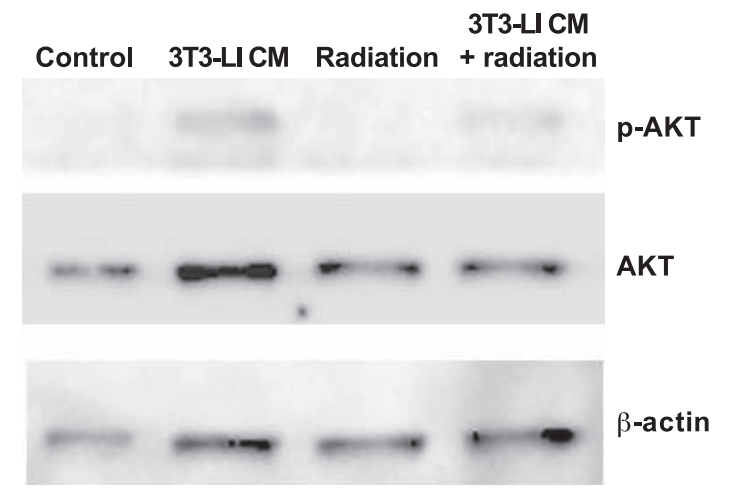

FIG. 3. Adipocyte-released factors protect B16-F10 melanocytes from fractionated radiation damage. Melanocytes were treated with 3T3-L1 CM, irradiated daily (2 Gy) for four consecutive days or received both treatments (3T3-L1 CM + radiation). At 12, 24, 48, 72 and $96 \mathrm{~h}$ after the first irradiation, cellular metabolic activity (panel A), catalase activity (panel B) and 3-nitrotirosine content (panel C) were determined. Results are represented as percentage of control. Bars show mean \pm SEM $\left(* P<0.05\right.$ vs. control treatment; ${ }^{*} P<0.05$ vs. inflammatory status and the adipokine and chemokine-rich environment that can protect malignant cells from radiation damage.

Oxidative stress has been proposed as a common link between obesity and cancer. The increased levels of circulating oxidants and ROS produced from peritumoral adipose tissue accelerate oxidative stress within tumor cells and might contribute to the increased risk for cancer progression in obese patients (22). ROS production also increases along the differentiation of 3T3-L1 cells into mature adipocytes (31), however, our results showed a higher antioxidant capacity of 3T3-L1 CM. Given the short half-life of radicals, this ROS-mediated effect might be less significant in our cell culture model. In fact, numerous 3T3L1 adipocyte-secreted proteins have implications in growth regulation and act as cell mitogens $(18,20,32)$. We believe these pro-proliferative and anti-apoptotic effects overcome the adipocyte-derived ROS.

Several signaling pathways involved in the radioprotective mechanisms of melanoma have been identified. MEK, ERK and PI3K-AKT cell survival cascades are known to play important roles in overcoming radiation-induced damage (33) and have implications for radioresistance mechanisms in cancer $(26,27)$. Blockage of these pathways has been shown to radiosensitize melanocytes to ionizing radiation (34). We have already demonstrated that 3T3-L1 $\mathrm{CM}$ is rich in numerous growth factors (20), such as insulinlike growth factor 1 (IGF-1) and hepatocyte growth factor (HGF), which have been linked to cancer radioresistance. Downregulation of insulin-like growth factor 1 (IGF-1) receptor in mouse melanoma B16-F1 cells improved radiosensitivity (35) and, in some malignancies, radioresistant phenotypes were aggravated by HGF (36). IGF-1 and HGF signaling activates PI3K-AKT pro-survival pathways (37). These findings led us to assume that the growth-factors present in the 3T3-L1 CM and the upregulation of the AKT signaling cascade are contributing to the radioresistant phenotype of B16-F10 cells observed in our results. Nevertheless, given the vast number of growth factors and bioactive molecules released by adipocytes, it is our belief that a "one mechanism fits all" approach is unlikely to exist and other signaling pathways certainly are co-activated and contribute to the enhanced radioresistance of melanoma.

Radiation also induces vascular damage that potentiates tumor hypoxia (38). In a recently published study, our group showed that adipocyte secretome enhances vasculogenic mimicry in B16-F10 cells (20). Therefore, reducing the vascular network dependence of the tumor for blood supply, which allied with the radiation-desensitizing effect, plays a cumulative role towards melanoma aggressiveness.

irradiation; $n=6$ ). Panel D: Immunoblot detection of AKT and Ser473 phosphorylated-AKT. Beta-actin was used as a loading control. A representative Western blot is shown. 
In summary, our results indicate that an environment rich in adipocyte-released factors contributes to the protection of melanocytes from radiation-induced oxidative stress and viability loss, circumventing the efficacy of radiation, with a contribution of adipocyte-released factors PI3K-AKT activation.

Although further in vivo studies are of paramount importance to elucidate the molecular players involved, the current study demonstrates the importance of taking into consideration the role of adiposity when planning radiotherapy regimens.

\section{ACKNOWLEDGMENTS}

We are immensely grateful to the personnel from "Serviço de Radioterapia, Centro Hospitalar de São João" for their assistance with the radiation procedures. We are also indebted to the "Fundação para a Ciência e a Tecnologia" and FEDER-COMPETE for financial support through the research unit PEst-OE/SAU/UI0038/2011, UID/BIM/04293/ 2013 and the PhD grant awarded to PC (no. SFRH/BD/80434/2011).

Received: June 22, 2016; accepted: December 30, 2016; published online: Month 00, 2017

\section{REFERENCES}

1. Geller AC, Clapp RW, Sober AJ, Gonsalves L, Mueller L, Christiansen CL, et al. Melanoma epidemic: an analysis of six decades of data from the connecticut tumor registry. J Clin Oncol 2013; 31:4172-8.

2. Siegel RL, Miller KD, Jemal A. Cancer statistics 2016. CA Cancer $\mathrm{J}$ Clin 2016; 66:7-30.

3. Greinert R, de Vries E, Erdmann F, Espina C, Auvinen A, Kesminiene A, et al. European Code against Cancer 4th Edition: Ultraviolet radiation and cancer. Cancer Epidemiol 2015; 39:S75S83.

4. Tuong W, Cheng LS, Armstrong AW. Melanoma: epidemiology, diagnosis, treatment, and outcomes. Dermatol Clin 2012; 30:11324.

5. Hubenak JR, Zhang Q, Branch CD, Kronowitz SJ. Mechanisms of injury to normal tissue after radiotherapy. Plast Reconstr Surg 2014; 133:49e-56e.

6. Riley PA. Free radicals in biology: oxidative stress and the effects of ionizing radiation. Int J Radiat Biol 1994; 65:27-33.

7. Khan N, Khan MK, Almasan A, Singh AD, MacKlis R. The evolving role of radiation therapy in the management of malignant melanoma. Int J Radiat Oncol Biol Phys 2011; 80:645-54.

8. Diehn M, Clarke MF. Cancer stem cells and radiotherapy: New insights into tumor radioresistance. J Natl Cancer Inst 2006; 98:1755-7.

9. Olivier KR, Schild SE, Morris CG, Brown PO, Markovic SN. A higher radiotherapy dose is associated with more durable palliation and longer survival in patients with metastatic melanoma. Cancer 2007; 110:1791-5.

10. Niezgoda A, Niezgoda P, Czajkowski R. Novel approaches to treatment of advanced melanoma: a review on targeted therapy and immunotherapy. Biomed Res Int 2015; 2015:851387.

11. Calle EE, Kaaks R. Overweight, obesity and cancer: epidemiological evidence and proposed mechanisms. Nat Rev Cancer 2004; 4:579-91.

12. Renehan AG, Tyson M, Egger M, Heller RF, Zwahlen M. Bodymass index and incidence of cancer: a systematic review and metaanalysis of prospective observational studies. Lancet 2008; 371:569-78.

13. Sergentanis TN, Antoniadis AG, Gogas HJ, Antonopoulos CN,
Adami HO, Ekbom A, et al. Obesity and risk of malignant melanoma: A meta-analysis of cohort and case-control studies. Eur J Cancer 2013; 49:642-57.

14. Wolin KY, Carson K, Colditz GA. Obesity and cancer. Oncologist 2010; 15:556-65.

15. Fang P, Tan KS, Troxel AB, Rengan R, Freedman G, Lin LL. High body mass index is associated with worse quality of life in breast cancer patients receiving radiotherapy. Breast Cancer Res Treat 2013; 141:125-33.

16. Strom SS, Kamat AM, Gruschkus SK, Gu Y, Wen S, Cheung MR, et al. Influence of obesity on biochemical and clinical failure after external-beam radiotherapy for localized prostate cancer. Cancer 2006; 107:631-9.

17. Karastergiou K, Mohamed-Ali V. The autocrine and paracrine roles of adipokines. Mol Cell Endocrinol 2010; 318:69-78.

18. Wang P, Mariman E, Keijer J, Bouwman F, Noben JP, Robben J, et al. Profiling of the secreted proteins during 3T3-L1 adipocyte differentiation leads to the identification of novel adipokines. Cell Mol Life Sci 2004; 61:2405-17.

19. Pandey V, Vijayakumar MV, Ajay AK, Malvi P, Bhat MK. Dietinduced obesity increases melanoma progression: involvement of Cav-1 and FASN. Int J Cancer 2012; 130:497-508.

20. Coelho P, Almeida J, Prudêncio C, Fernandes R, Soares R. Effect of Adipocyte Secretome in Melanoma Progression and Vasculogenic Mimicry. J Cell Biochem 2016; 117:1697-706.

21. Patwardhan RS, Sharma D, Checker R, Thoh M, Sandur SK. Spatio-temporal changes in glutathione and thioredoxin redox couples during ionizing radiation-induced oxidative stress regulate tumor radio-resistance. Free Radic Res 2015; 49:1218-32.

22. Matsuda M, Shimomura I. Increased oxidative stress in obesity: Implications for metabolic syndrome, diabetes, hypertension, dyslipidemia, atherosclerosis, and cancer. Obes Res Clin Pract 2013; 7:1-12.

23. Teixeira D, Prudencio C, Vieira M. Development of a new HPLCbased method for 3-nitrotyrosine quantification in different biological matrices. J Chromatogr B Analyt Technol Biomed Life Sci 2017; 1046:48-57.

24. Ayala A, Muñoz MF, Argüelles S. Lipid peroxidation: production, metabolism, and signaling mechanisms of malondialdehyde and 4hydroxy-2-nonenal. Oxid Med Cell Longev 2014; 2014:360438.

25. Giaid A, Lehnert SM, Chehayeb B, Chehayeb D, Kaplan I, Shenouda G. Inducible nitric oxide synthase and nitrotyrosine in mice with radiation-induced lung damage. Am J Clin Oncol 2003; 26:e67-e72.

26. Bussink J, van der Kogel AJ, Kaanders JH. Activation of the PI3K/AKT pathway and implications for radioresistance mechanisms in head and neck cancer. Lancet Oncol 2008; 9:288-96.

27. Li H-F, Kim J-S, Waldman T. Radiation-induced Akt activation modulates radioresistance in human glioblastoma cells. Radiat Oncol 2009; 4:43.

28. Gorayski P, Burmeister B, Foote M. Radiotherapy for cutaneous melanoma: current and future applications. 2015; 11:525-34.

29. Barker HE, Paget JTE, Khan AA, Harrington KJ. The tumour microenvironment after radiotherapy: mechanisms of resistance and recurrence. Nat Rev Cancer 2015; 15:409-25.

30. Wong JR, Gao Z, Merrick S, Wilson P, Uematsu M, Woo K, et al. Potential for higher treatment failure in obese patients: correlation of elevated body mass index and increased daily prostate deviations from the radiation beam isocenters in an analysis of 1,465 computed tomographic images. Int J Radiat Oncol Biol Phys 2009; 75:49-55.

31. Furukawa S, Fujita T, Shimabukuro M, Iwaki M, Yamada Y, Nakajima $\mathrm{Y}$, et al. Increased oxidative stress in obesity and its impact on metabolic syndrome. J Clin Invest 2004; 114:1752-61.

32. Kratchmarova I. A Proteomic approach for identification of secreted proteins during the differentiation of 3T3-L1 preadipocytes to adipocytes. Mol Cell Proteomics 2002; 1:213-22. 
33. Johnson GE, Ivanov VN, Hei TK. Radiosensitization of melanoma cells through combined inhibition of protein regulators of cell survival. Apoptosis 2008; 13:790-802.

34. Eder S, Lamkowski A, Priller M, Port M, Steinestel K. Radiosensitization and downregulation of heterogeneous nuclear ribonucleoprotein K (hnRNP K) upon inhibition of mitogen/ extracellular signal-regulated kinase (MEK) in malignant melanoma cells. Oncotarget 2015; 6:17178-91.

35. Macaulay VM, Salisbury AJ, Bohula EA, Playford MP, Smorodinsky NI, Shiloh Y. Downregulation of the type 1 insulin-like growth factor receptor in mouse melanoma cells is associated with enhanced radiosensitivity and impaired activation of Atm kinase. Oncogene 2001; 20:4029-40.

36. De Bacco F, Luraghi P, Medico E, Reato G, Girolami F, Perera T, et al. Induction of MET by ionizing radiation and its role in radioresistance and invasive growth of cancer. J Natl Cancer Inst 2011; 103:645-61.

37. Jung HJ, Suh Y. Regulation of IGF-1 signaling by microRNAs. Front Genet 2015; 5:1-14.

38. Rockwell S, Dobrucki IT, Kim EY, Marrison ST, Vu VT. Hypoxia and radiation therapy: past history, ongoing research, and future promise. Curr Mol Med 2009; 9:442-58. 